\title{
Violência doméstica: A importância da formação do Cirurgião-Dentista frente a esse
}

\author{
agravo \\ Domestic violence: The importance of the training of the Dental Surgeon against this condition \\ Violencia doméstica: La importancia de la formación del Cirujano-Dentista frente a ese agravio
}

Recebido: 05/04/2021 | Revisado: 10/04/2021 | Aceito: 14/04/2021 | Publicado: 26/04/2021

\author{
Anayla Oliveira da Silva \\ ORCID: https://orcid.org/0000-0002-8112-1650 \\ Universidade Potiguar, Brasil \\ E-mail: anaylasilva@hotmail.com \\ Maria da Conceição Lara Costa Silva \\ ORCID: https://orcid.org/0000-0002-7263-9669 \\ Universidade Potiguar, Brasil \\ E-mail: mlaracosta_@hotmail.com \\ Amanda Barbosa de Godoy \\ ORCID: https://orcid.org/0000-0002-8798-9725 \\ Universidade Potiguar, Brasil \\ E-mail: amandagodoy99@gmail.com \\ Luanna Maria Rocha Caldas da Silva \\ ORCID: https://orcid.org/0000-0001-6925-9721 \\ Universidade Potiguar, Brasil \\ E-mail: luannarcaldas@gmail.com \\ Ana Larissa Fernandes de Holanda Soares \\ ORCID: https://orcid.org/0000-0002-3740-7862 \\ Universidade Potiguar, Brasil \\ E-mail: ana.larissa@unp.br
}

\begin{abstract}
Resumo
Violência doméstica é qualquer ação ou omissão que cause morte, lesão, sofrimento físico, sexual ou psicológico e dano moral ou patrimonial. O cirurgião-dentista é um dos profissionais que possui maior contato com estas vítimas, devido ao maior comprometimento na região orofacial dos pacientes violentados. O objetivo do presente trabalho é discutir a importância da formação acadêmica do cirurgião-dentista frente a violência doméstica. A pesquisa foi realizada com alunos do curso de odontologia de uma universidade de Natal/RN, do nono período. Para colhimento e validação dos dados, questionários foram aplicados de forma individual e reservada para cada um. Participaram da pesquisa 57 alunos. Afirmaram que o cirurgião-dentista deve saber e ter a responsabilidade na identificação e no diagnóstico de casos de violência doméstica, 54 (94,7\%) dos entrevistados. Quanto às providências que devem ser tomadas, pelo profissional, a escolha por "notificar a agressão" foi a mais relatada. Em relação ao tema ter sido abordado no curso, $30(52,6 \%)$ estudantes relataram ter tido contato com o tema estudado, desses, a maior parte dos entrevistados $16(53,3 \%)$, responderam não lembrar ao certo quando isso aconteceu. Com esta pesquisa, concluiu-se que há necessidade de uma maior abordagem sobre esta temática por parte dos professores que ministram as disciplinas básicas da grade curricular, pois, ainda que os participantes tenham respondido de forma positiva aos questionamentos, notou-se uma insegurança e deficiência perante o tema proposto.
\end{abstract}

Palavras-chave: Violência doméstica; Cirurgião-dentista; Notificação compulsória.

\begin{abstract}
Domestic violence is any act or omission that causes death, injury, physical, sexual or psychological suffering, and moral or property damage. The dental surgeon is one of the professionals who has greater contact with these victims, due to the greater involvement in the orofacial region of the abused patients. The objective of the present study is to discuss the importance of the academic training of dental surgeons in the face of domestic violence. The research was carried out with students of the dentistry course of a university of Natal/RN, of the ninth period. For data collection and validation, questionnaires were applied individually and reserved for each one. 57 students participated in the study. They stated that the dental surgeon should know and be responsible for identifying and diagnosing cases of domestic violence, $54(94.7 \%)$ of the interviewees. As for the measures that should be taken, by the professional, the choice to "notify the aggression" was the most reported. In relation to the subject being addressed in the course, 30 (52.6\%) students reported having had contact with the subject studied, of these, most of the interviewees $16(53.3 \%)$, answered not to remember when this happened. With this research, it was concluded that there is a need for a greater approach on this subject by the teachers who teach the basic disciplines of the curriculum grid, because, although the
\end{abstract}


participants responded positively to the questions, an insecurity and deficiency in relation to the proposed theme has been noted.

Keywords: Domestic violence; Dental surgeon; Compulsory notification.

\begin{abstract}
Resumen
Violencia doméstica es cualquier acción u omisión que cause muerte, lesiones, sufrimiento físico, sexual o psicológico y daño moral o patrimonial. El cirujano-dentista es uno de los profesionales que posee mayor contacto con estas víctimas, debido al mayor comprometimiento en la región orofacial de los pacientes violentados. El objetivo del presente trabajo es discutir la importancia de la formación académica del cirujano-dentista frente a la violencia doméstica. La investigación fue realizada con alumnos del curso de odontología de una universidad de Natal/RN, del noveno período. Para recopilar y validar los datos, se aplicaron cuestionarios de forma individual y reservada para cada uno. Participaron de la encuesta 57 alumnos. Afirmaron que el cirujano-dentista debe saber y tener la responsabilidad en la identificación y en el diagnóstico de casos de violencia doméstica, 54 (94,7\%) de los entrevistados. En cuanto a las medidas que deben ser tomadas, por el profesional, la elección por "notificar la agresión" fue la más informada. En relación con el tema que se abordó en el curso, $30(52,6 \%)$ estudiantes informaron haber tenido contacto con el tema estudiado, de estos, la mayoría de los encuestados 16 (53,3\%), respondieron no recordar exactamente cuando esto sucedió. Con esta investigación, se concluyó que hay necesidad de un mayor abordaje sobre esta temática por parte de los profesores que ministran las disciplinas básicas de la rejilla curricular, pues, aunque los participantes hayan respondido de forma positiva a los cuestionamientos, se ha observado una inseguridad y una discapacidad ante el tema propuesto.
\end{abstract}

Palabras clave: Violencia doméstica; Cirujano-dentista; Notificación obligatoria.

\title{
1. Introdução
}

A violência doméstica é definida como violência física ou emocional contra parceiro íntimo, idosos, crianças e animais de estimação (Mazza, Marano, Lai, Janiri \& Sani, 2020). Segundo a Organização Mundial da Saúde a violência doméstica é uma forma agressiva e opressora de violência interpessoal, na qual o agressor usa táticas de controle para abusar emocionalmente, fisicamente, sexualmente ou economicamente de um membro da família ou parceiro íntimo (OMS, 2018). A unidade doméstica na qual se pratica essa violência é compreendida como o espaço de convívio permanente de pessoas, com ou sem vínculo familiar (Art. 5º , Lei 11.340/06, 2006).

A violência doméstica pode ser por meio da violência física, sexual e ou psicológica. A violência física é entendida como qualquer conduta que ofenda a integridade ou saúde corporal da mulher, já a violência psicológica é qualquer conduta que cause danos emocionais, diminuição da autoestima, prejudique e perturbe o pleno desenvolvimento da mulher; ou vise degradar e ou controlar suas ações, comportamentos, crenças e decisões. Violência sexual é tida como qualquer conduta que constranja a vítima e a obrigue a manter ou a participar de relação sexual não desejada mediante intimidação, ameaça, coação ou uso da força (LEI No 11.340, 2006).

A violência contra a mulher constitui uma das formas de violação dos direitos humanos. O feminicídio, é uma das consequências da violência doméstica que afetam diversos indivíduos (Art. 6º Lei 11.340/06, 2015). Em longo prazo, a violência doméstica é um fator de risco para diversas condições de saúde mental, como transtornos de humor, alimentares, ansiedade e transtorno de estresse pós-traumático, gerando abuso de álcool e/ou substâncias (El-serag \& Thurston, 2020).

Presenciar ou participar ativamente desse tipo de violência durante a juventude, aumenta as chances de automutilação ou ideação suicida no futuro (Mazza; Clarke, 2020, 2020). A exposição à violência familiar aumenta as chances de um indivíduo perpetuar ações violentas contra seus parceiros íntimos e/ou familiares, desenvolvendo mudanças comportamentais significativas (Igram, Espelage, Davis \& Merrin, 2020).

Com a pandemia do novo coronavírus, a vida dos indivíduos transformou-se devido as medidas de contenção e proteção da Covid-19, o que modificou a estrutura familiar, gerando alguns problemas e tensões. Com isso, o lar corre o risco de se tornar um local muito perigoso para as vítimas da violência doméstica (Mazza, Marano, Lai, Janiri \& Sani, 2020). Para as famílias que moram em casas pequenas ou sem espaços abertos, o estresse é exacerbado. A suspenção das aulas presenciais pode ainda aumentar a exposição de crianças e adolescentes à violência doméstica com consequências diretas à sua saúde 
física e mental (Cluver et al., 2020).

No Brasil, a violência doméstica é considerada um agravo de notificação compulsória. O agravo se dá devido aos danos gerados à integridade física ou mental de um indivíduo e é de notificação compulsória, pois é realizada pelos profissionais de saúde ou responsáveis pelos estabelecimentos de saúde, públicos ou privados (Portaria № 204, 2016).

Com isso, a importância da formação acadêmica dos profissionais de saúde voltada para a detecção precoce desse agravo, gera um sistema bem estruturado de educação e apoio as vítimas. As universidades públicas e privadas de todo o país deveriam oferecer treinamento especial a esses profissionais para identificar, apoiar e tratar pacientes em violência doméstica, o que seria de suma importância para a sociedade e para a diminuição dos casos de subnotificação (Usta; Sardinha, 2012, 2018).

As lesões decorrentes da violência física podem atingir a face e seus tecidos moles, ocasionando também fraturas nos ossos faciais e no complexo maxilomandibular (Ferreira; Ungari, 2014, 2012). Esses dados, expressam a importância do profissional de saúde cirurgião-dentista no diagnóstico dessas lesões, as quais, na grande maioria das vezes, passam despercebidas por ocasião da anamnese e do exame clínico em decorrência desse profissional desconhecer os sinais básicos para seu diagnóstico precoce.

Nessa perspectiva, são importantes os estudos que tragam ao debate essa realidade e que destaquem a importância da discussão sobre esse tema ainda na graduação para que os profissionais sejam adequadamente preparados e estimulados a enfrentarem esse agravo, auxiliando na notificação dos casos e na diminuição das estatísticas.

O objetivo do presente trabalho foi discutir a formação acadêmica do cirurgião-dentista e a sua importância na atuação desse futuro profissional diante do diagnóstico de caso de violência doméstica. Além disso, identificar em quais momentos os referenciais teóricos e práticos foram abordados na graduação dos alunos do curso de odontologia de uma universidade localizada em Natal/RN e descrever a percepção e o conhecimento do protocolo a ser realizado frente à violência doméstica entre os alunos.

\section{Metodologia}

Estudo transversal, descritivo, exploratório e quantitativo, realizado em uma universidade de Natal localizada no Rio Grande do Norte (RN), no ano de 2018. Trata-se de um censo, na qual a pesquisa foi composta por todos os alunos regularmente matriculados em uma das turmas do nono semestre do curso de odontologia. Os critérios de inclusão foram: ser aluno da universidade, cursar a nona série e estar matriculado na referida turma. Os critérios de exclusão foram os alunos não estarem presentes no momento da coleta dos dados, não querer participar da pesquisa e os que levaram o questionário para casa.

A operacionalização do estudo foi realizada com o contato direto na coordenação do curso, para apresentação do projeto e solicitação de autorização. Após aprovação do comitê de ética, foi aplicado de forma individual um questionário semiestruturado com perguntas abertas, referentes às características gerais dos participantes, assim como questionamentos relacionados a temática do estudo (período em que foi abordado o tema na graduação; metodologias teóricas e práticas utilizadas para trabalhar o assunto; percepção do graduando sobre o papel do cirurgião-dentista frente à violência doméstica).

Os dados foram analisados de forma descritiva, a partir de tabelas e gráficos. Esse artigo é parte integrante da pesquisa "Violência doméstica: a importância da formação do cirurgião-dentista frente a esse agravo", aprovada pelo Comitê de ética e pesquisa da universidade, com registro do parecer $\mathrm{N}^{\circ}$ 2.583.474. A autorização para participar do estudo, foi realizada por meio de termo de consentimento livre e esclarecido (TCLE), emitido e assinado em duas vias, ficando uma via com o participante e a outra com o aplicador do questionário, os participantes foram informados das razões do estudo e esclarecidos que poderiam desistir de participar em qualquer etapa da pesquisa sem prejuízos. Foram informados, também, que os dados 
seriam mantidos em sigilo e a identidade dos indivíduos resguardada.

Toda pesquisa com seres humanos envolve risco em tipos e gradações variados. O estudo vislumbrou como possível risco emocional o constrangimento dos participantes. Dessa forma, para minimizar esse risco, os questionários foram aplicados individualmente e em um local reservado.

O benefício foi identificar o conhecimento dos graduandos sobre a importância do cirurgião-dentista no diagnóstico de casos de violência doméstica.

\section{Resultados}

De acordo com os resultados obtidos, dos 60 questionários aplicados, apenas 1 retornou sem respostas e 2 foram anulados em razão dos respectivos participantes os terem levado para suas casas, totalizando assim 57 participantes ao final. A amostra foi composta por $23(40,3 \%)$ estudantes do sexo masculino e $34(59,6 \%)$ de estudantes do sexo feminino. A média de idade foi de 32,5 anos (Desvio-padrão $=5.7$ ).

Em relação a percepção dos alunos quanto ao futuro contato com pacientes vítimas de violência doméstica, no exercício da sua prática clínica, dos 57 questionários aplicados, apenas 1(2\%) entrevistado relatou achar que não terá contato com casos de violência doméstica, em contrapartida 56 (98\%) dos entrevistados responderam achar que terão sim contato com esse tipo de paciente.

No Gráfico 1, obteve-se os dados referente ao questionamento da responsabilidade de notificação do agravo.

Gráfico 1 - Respostas sobre a responsabilidade do ato de notificação.

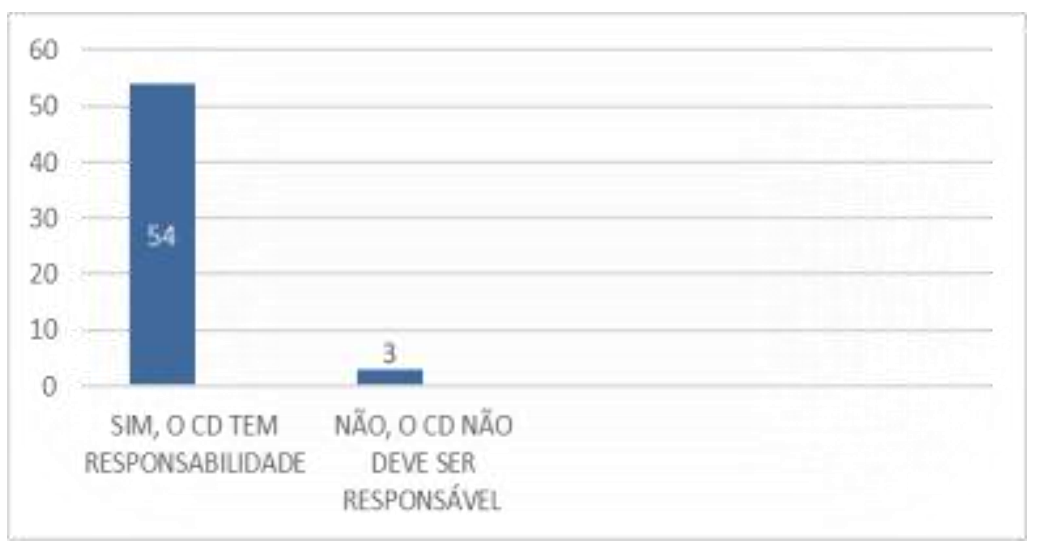

Fonte: Questionários aplicados pelos autores (Natal/2018).

Das respostas obtidas com esse questionamento, 54 (94,7\%) dos entrevistados afirmaram que o cirurgião dentista deve ter responsabilidade na identificação e no diagnóstico de casos de violência doméstica, havendo apenas $3(5,3 \%)$ entrevistados em discordância.

Nos dados obtidos no Gráfico 2, admitia aos entrevistados mais de uma opção de escolha no que se refere a situações que se caracterizam como violência doméstica. 
Gráfico 2 - Respostas sobre quais condutas são consideradas violência doméstica por parte dos estudantes.

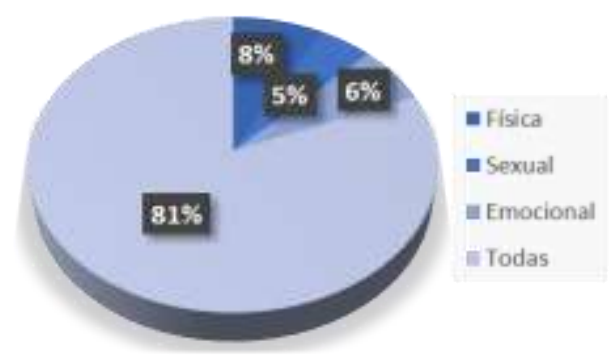

Fonte: Questionários aplicados pelos autores (Natal/2018).

Dentre as alternativas, violência física, sexual, emocional e a opção "todas" foram questionadas. Mas o item "todas" foi o de maior prevalência com (81\%). Já no Gráfico 3, os dados coletados são sobre as possíveis formas do cirurgião-dentista fazer o diagnóstico da violência doméstica.

Gráfico 3 - Respostas sobre a forma do cirurgião-dentista realizar o diagnóstico de violência doméstica.

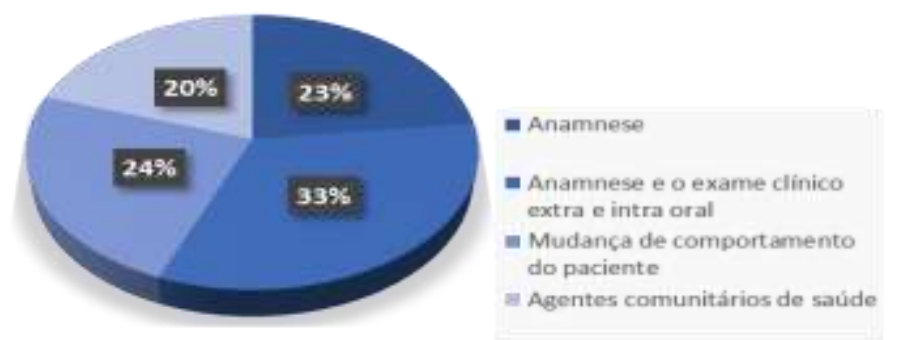

Fonte: Questionários aplicados pelos autores (Natal/2018).

Sobre a forma de diagnóstico, os dados foram bastante divergentes, mostrando uma falta de padronização desses futuros profissionais, quanto ao melhor diagnóstico nos casos de violência doméstica.

Quanto às providências que devem ser tomadas pelo cirurgião-dentista, diante de um caso de violência doméstica, o gráfico 4 mostra diferentes possibilidades de conduta diante desses casos.

Gráfico 4 - Condutas frente à um caso de violência doméstica.

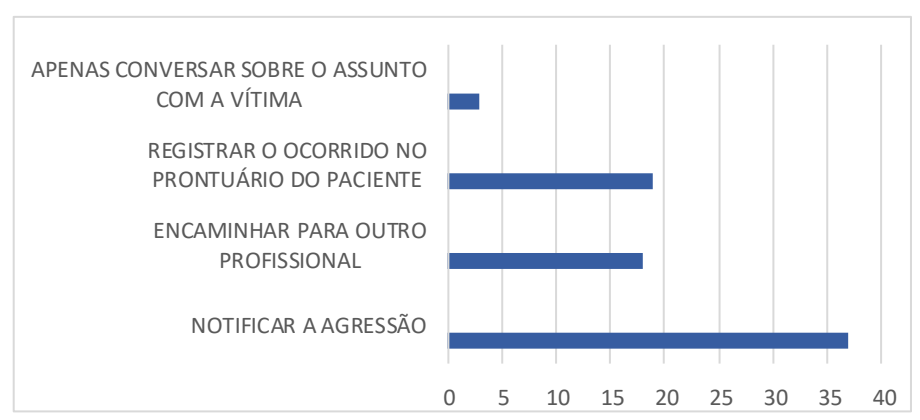

Fonte: Questionários aplicados pelos autores (Natal/2018). 
De acordo com os dados obtidos, a escolha por "notificar a agressão" predominou entre os entrevistados, 37, havendo um distanciamento desse resultado com "encaminhar para outro profissional" 18 e "registrar o ocorrido no prontuário do paciente" 19. A conduta de "apenas conversar sobre o assunto com a vítima" foi a menos citada pelos entrevistados, 3.

Sobre a presença na grade curricular do curso de graduação de odontologia de disciplinas que abordaram a temática da violência doméstica, $30(52,6 \%)$ estudantes disseram ter tido contato durante o curso, enquanto os outros 27 (47,3\%) negaram.

Levando em consideração os que disseram ter tido contato com a temática aqui estudada, durante a graduação, a maior parte desses entrevistados 16 (53,3\%), responderam não lembrar do período do curso onde isso aconteceu ou não sabiam especificar $14(46,6 \%)$.

Entre as metodologias utilizadas pela universidade para trabalhar a violência doméstica em sala de aula, dos entrevistados, 38 (66,7\%) mencionaram ter tido contato com a temática por meio de "aula teórica", outros 10 (16,7\%) por meio de "mapa conceitual" e 9 (16,6\%), relataram "outra" onde a última não foi especificada por eles.

Quanto à capacidade de lidar futuramente com casos de violência doméstica, o Gráfico 5 ilustra como se sentem os profissionais perante esse questionamento.

Gráfico 5 - Capacidade de lidar com casos de violência doméstica.

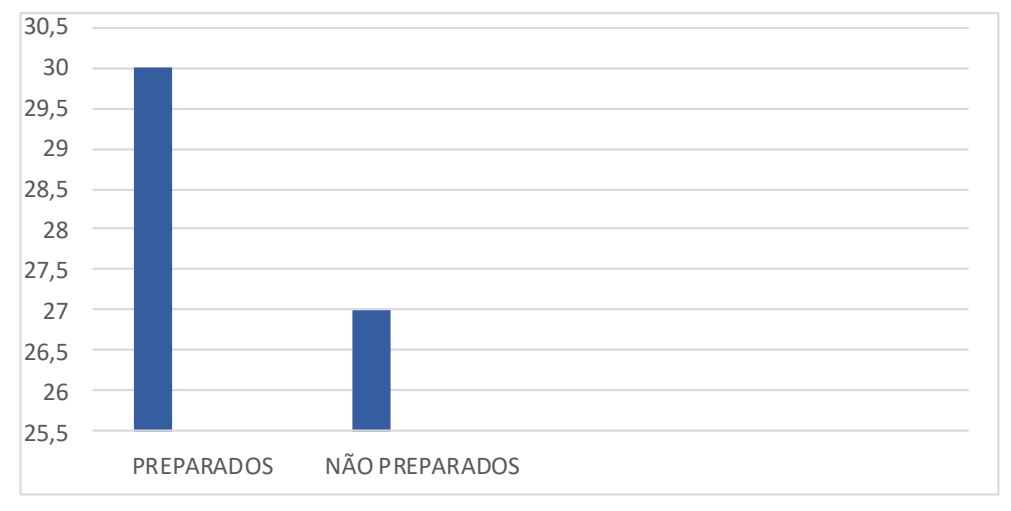

Fonte: Questionários aplicados pelos autores (Natal/2018).

$\mathrm{Na}$ análise do gráfico, constata-se que $30(52,6 \%)$ dos entrevistados afirmaram se sentir preparados para lidar com esta situação, contra outros $27(47,4 \%)$ que disseram não estarem preparados.

\section{Discussão}

A violência tem se mostrado um grande problema de saúde pública no mundo, ao afetar diretamente a integridade da sociedade, das famílias e dos indivíduos tanto fisicamente quanto mentalmente (Muszkat \& Muszkat, 2018).

A Organização Mundial da Saúde (OMS), estima que 1 em cada 3 (35\%) das mulheres sofrem violência doméstica pelo menos uma vez durante a vida, e que aproximadamente $80 \%$ dos profissionais de saúde nunca receberam treinamento sobre o manejo desses pacientes (OPAS, 2017). No Brasil, entre 2013 e 2018, a taxa de homicídios domiciliares aumentou $8,3 \%$. o percentual de mulheres que sofrem a violência dentro da residência é 2,7 maior do que o de homens, o que amplia e reflete a dimensão da violência de gênero e, em particular, do feminicídio (IBGE, 2018).

Costa, Carvalho, Santana, Silva e Silva (2010) destacaram que o cirurgião-dentista é um dos profissionais que possui maior contato com pacientes vítimas de violência doméstica, sejam crianças, adultos ou idosos, haja vista que 50\% das lesões decorrentes de violência, se referem a traumas orofaciais. No presente trabalho, a maioria dos entrevistados respondeu que 
previam a possibilidade futura do contato com algum caso de violência doméstica na sua prática clínica, o que nos leva a confirmar a importância e a responsabilidade desses profissionais diante do problema estudado.

A notificação compulsória é obrigatória para os profissionais de saúde ou responsáveis pelos serviços públicos e privados de saúde, que prestam assistência ao paciente, em conformidade com o art. $8^{\circ}$ da Lei $n^{\circ} 6.259$, de 30 de outubro de 1975. A notificação não é uma denúncia policial e deve ser feita independente da permissão da vítima, nos casos em que houver indícios ou confirmação de violência doméstica, para que as providências cabíveis sejam tomadas e registradas no Sistema de Informação de Agravos de Notificação (SINAN), que tem por objetivo subsidiar a elaboração e execução de políticas públicas para o enfrentamento da violência no país (Brasil, 2019).

A portaria no 204, de 17 de fevereiro de 2016 atualizou a Lista Nacional de Notificação Compulsória de doenças, agravos e eventos de saúde pública nos serviços de saúde em todo o território nacional.

Apesar dos avanços que tange a legislação da notificação, ainda se trata de uma ferramenta invisível na rotina dos profissionais da saúde. Segundo a pesquisa realizada por Sousa et al (2015), observou-se o não conhecimento da ficha de notificação compulsória nos casos de violência contra mulher por parte dos profissionais, não estando claro para eles quem seriam os profissionais responsáveis pelo preenchimento. Com isso, tem-se a subnotificação que é uma situação persistente no Brasil e que está relacionada à falta de informações técnicas e científicas do assunto (Garbin, Dias, Rovida \& Garbin, 2017). Isso explicita a falta de normatização, tanto no preenchimento da ficha de notificação, quanto da notificação às autoridades policiais, inserida na Lei em 2019 (Feitoza \& Sampaio, 2020).

A subnotificação proveniente desses casos, se dá, principalmente, pela vergonha, constrangimento, estigma social, medo e dependência do agressor, as vítimas se sentem acuadas e muitas vezes não estão dispostas a revelar seus traumas a outras pessoas (Kaur \& Garg; OMS, 2008, 2018).

De acordo com a natureza dos atos violentos, $46(81 \%)$ dos estudantes de odontologia entrevistados, relataram considerar como violência doméstica, a violência física, a emocional e a sexual. Em 2007, em seu artigo, Dahlberg e Krug também relacionaram essas categorias com a violência doméstica, o que corrobora com os achados aqui encontrados.

Nos resultados deste estudo, os dados demostraram que 19 (33\%) dos participantes escolheram como melhor maneira para fazer o diagnóstico da violência doméstica, a anamnese e o exame clínico extra e intraoral. Além disso, diante do diagnóstico, o procedimento a ser realizado, pela maioria dos estudantes, foi a notificação, o qual está totalmente coerente com o preconizado na literatura sobre o tema.

Quanto à abordagem durante o curso, 30 (52,6\%) entrevistados disseram ter tido, ao longo de sua formação acadêmica, contato com essa temática. A formação profissional é importante, uma vez que, ao inserir o assunto violência doméstica no currículo dos cursos de graduação, estimula os graduandos a desenvolverem a consciência crítica das forças sociais maiores que afetam a vida e a saúde e a reconhecer seu potencial como membro de uma comunidade que se posiciona contra a violência e que cumpre o seu papel com responsabilidade (Souza et al, 2013).

Jaramillo e Uribe (2006), defenderam que grande parte das disciplinas da área da saúde não contemplam, em seus currículos e nem em seus programas de educação continuada, a formação nos aspectos relacionados com a violência doméstica e, por isso, os alunos e futuros profissionais não se encontram preparados para oferecer uma atenção que seja efetiva na saúde da mulher vítima de violência.

$\mathrm{Na}$ presente pesquisa, a maior parte dos entrevistados informou estar preparada para encarar a situação, caso seja necessário, em sua prática profissional. Essa contradição pode ser justificada pela hipótese de que os alunos, por estarem no último período do curso, podem ter ampliado seus conhecimentos o longo da graduação 
A implementação da educação digital no dia a dia dos alunos ajuda também a ampliar e melhorar a gestão sobre diversos assuntos, entre eles, a violência doméstica, melhorando a relação custo-eficácia dos programas educacionais (Runyan; Shefet, 2005, 2007).

Em relação às limitações deste trabalho, é possível destacar o fato de ser um estudo transversal e não possibilitar fechar causalidade, além do número pequeno de amostras. Os dados são válidos para os alunos entrevistados dentro da instituição pesquisada, não representando de forma alguma a população de estudantes em geral. Em contrapartida, o estudo tem grande importância por debater um tema preocupante e pouco explorado na literatura científica, haja vista a limitação de estudos publicados, principalmente relacionados com estudantes ou profissionais de odontologia.

Independentemente do cenário caótico proporcionado pela Covid-19, dois anos após os dados coletados da pesquisa, a violência doméstica continua sendo uma questão de saúde pública. (Pang \& Thomas, 2020). Fica evidente a necessidade de futuras pesquisas sobre a violência doméstica, principalmente frente a pandemia de Covid-19, e o papel do profissional de saúde no seu diagnóstico precoce e encaminhamento adequado, dessa forma, será possível atuar de maneira responsável como coordenador e ordenador do cuidado.

\section{Conclusão}

Portanto, a pesquisa mostrou a importância dos profissionais de saúde no correto diagnóstico frente a quadros de violência doméstica e o papel de destaque que a formação acadêmica exerce nesse contexto.

A maioria dos estudantes responderam de forma correta sobre a principal conduta a ser realizada, reconhecendo a importância da notificação, diante de um diagnóstico de violência doméstica, o que vai a encontro com estudos anteriores. Torna-se importante um aprofundamento do tema no currículo da graduação pelos professores e gestores das instituições de ensino superior, para que a atuação profissional diante da violência doméstica seja mais efetiva e contribua para um melhor enfretamento desse quadro na sociedade.

\section{Referências}

Abdo, C., Miranda, E. P., Santos, C. S., Júnior, J. B., \& Bernardo, W. M. (2020). Domestic violence and substance abuse during COVID19: A systematic review. Indian journal of psychiatry.

Bott, S., Guedes, A., Ruiz-Celis, A. P., \& Mendoza, J. A. (2019). Intimate partner violence in the Americas: a systematic review and reanalysis of national prevalence estimates. Revista panamericana de salud publica. Pan American journal of public health.

Brasil, (2016). Portaria n 204, de 17 de fevereiro de 2016. Brasília: Ministério da Saúde, 2003. Lei n. 10.778, de 24 de novembro de 2003.

Brasil, (2019). Altera a Lei ${ }^{\circ} 10.778$, de 24 de novembro de 2003, para dispor sobre a notificação compulsória dos casos de suspeita de violência contra a mulher. Lei ${ }^{\circ} 13.931$, de 10 de dezembro. Brasília.

Clarke, A., Olive, P., Akooji, N., \& Whittaker, K. (2020). Exposição à violência e vulnerabilidade dos jovens, saúde física e mental. Int J Saúde Pública.

Cluver, L., Lachman, J. M., Sherr, L., Wessels, I., Krug, E., Rakotomalala, S., Blight, S., Hillis, S., Bachman, G., Green, O., Butchart, A., Tomlinson, M., Ward, C. L., Dúvida, J., \& McDonald, K. (2020). Paternidade em um tempo de COVID-19. Lanceta.

Costa, M. C., Carvalho, R. C., Santana, M., A., Silva, L., M., \& Silva, M. R. (2010). Evaluation of the National Program of Integrated and Referential Actions (PAIR) to confront the child and adolescents sexual violence, in Feira de Santana, Bahia State, Brazil. Ciênc Saúde Col.

Divakar, U., Nazeha, N., Posadzki, P., Jarbrink, K., Bajpai, R., Ho, A., Campbell, J., Feder, G., \& Car, J. (2019). Digital Education of Health Professionals on the Management of Domestic Violence: Systematic Review and Meta-Analysis by the Digital Health Education Collaboration. Journal of medical Internet research.

El-Serag, R., \& Thurston, R. C. (2020). Assuntos do coração e da mente: violência interpessoal e doenças cardiovasculares em mulheres. J Am Heart Assoc.

Feitoza, D. C., \& Sampaio, J., M., C. (2020). A notificação compulsória em situação de violência contra a mulher prevista nos códigos de ética da enfermagem, medicina, psicologia e odontologia. Centro Universitário de Brasília Faculdade de Ciências da Educação.

Ferreira, M. C., Batista, A. M., Ferreira, F. O., Ramos-Jorge, M. L., \& Marques, L. S. (2014). Pattern of oral-maxillofacial trauma stemming from interpersonal physical violence and determinant factors. Dent Traumatol. 
Garbin, C., A. S., Dias, I. A., Rovida, T. A. S., \& Garbin, A. J. I. (2017). Desafios do profissional de saúde na notificação da violência: obrigatoriedade, efetivação e Arch Health Invest. Ciênc. saúde coletiva.

IBGE, (2018). Diretoria de Pesquisas. Coordenação de População e Indicadores Sociais. Gerência de Estudos e Análises da Dinâmica Demográfica e MS/SVS/CGIAE Sistema de Informações sobre Mortalidade - SIM.

Ingram, K. M., Espelage, D. L., Davis, J. P., \& Merrin, G. J. (2020). Violência Familiar, Agressão de Irmãos e Pares Durante a Adolescência: Associações com Resultados de Saúde Comportamental. Frente. Psiquiatria.

Jaramillo, D., E., \& Uribe, T. M. (2006). Rol del personal em La atención a lãs mujeres maltratadas.Invest Educ Enferm.

Kaur, R., \& Garg, S. (2008). Enfrentando a violência doméstica contra as mulheres: uma agenda inacabada. Indian J Community Med.

Lai, Y. L., \& Ren, L. H. N., (2018). Os efeitos da violência doméstica sobre a má conduta violenta na prisão, o estado de saúde e a necessidade de assistência pós-libertação entre infratores da legislação antidrogas em Taiwan. Int J Ofensor Ther Comp Criminol.

Mazza, M., Marano, G., Lai, C., Janiri, L., \& Sani, G. (2020). Perigo em perigo: Danger in danger: Interpersonal violence during COVID-19 quarantine. Psychiatry Res. 2020; 289: 113046.

Ministério da Saúde (2016). Portaria No 204 de 17 de fevereiro de 2016. Define a Lista Nacional de Notificação Compulsória de doenças, agravos e eventos de saúde pública nos serviços de saúde. Brasília, DF.

Muszkat, M., Muszkat, S. (2016). Violência Familiar. Blucher.

Organização Mundial da Saúde, (2018). Compreendendo e abordando a violência contra as mulheres. Violência por parceiro íntimo.

Organização Pan-Americana da Saúde (OPAS), (2017). Folha informativa - Violência contra as mulheres, Brasília, DF.

Pang, L. H. G. (2020). Thomas SJ. Exposição à violência doméstica durante a adolescência: estratégias de enfrentamento e estilos de apego como moderadores iniciais e sua relação com o funcionamento durante a vida adulta. J Child Adolesc Trauma.

Runyan, C. W., Gunther-Mohr, C., Orton, S., Umble, K., Martin, S., L., \& Coyne-Beasley, T. (2005). PREVENT: um programa da Iniciativa Nacional de Treinamento em Prevenção de Lesões e Violência. Am J Prev Med.

Sardinha, L., Nájera, \& Catalán, H. E. (2018) Atitudes em relação à violência doméstica em 49 países de renda média baixa: uma análise de gênero da prevalência de correlações em nível de país. PLOS ONE.

Shefet, D., Dascal-Weichhendler, H., Rubin, O, Pessach, N., Itzik, D., Benita, S., \& Ziv, A. (2007). Violência doméstica: um programa educacional baseado em simulação nacional para melhorar o conhecimento, habilidades e taxas de detecção dos médicos. Med Teach.

Sousa, M. H., et al. (2015). Preenchimento da notificação compulsória em serviços de saúde que atendem mulheres que sofrem violência sexual. Revista brasileira epidemiologia.

Souza, E. R., Ribeiro, A. P., Penna, L. H. G., Ferreira, A. L., Santos, N. C., \& Tavares, C. M. M. (2013). Domestic violence in the conception of health profissionals trainers. Ciênc saúde coletiva.

Taylor, J., Bradbury-Jones, C., Lazenbatt, A., \& Soliman, F. (2016). Maus-tratos infantis: Caminho para condições crônicas e de longo prazo. J Public Health $(\mathrm{Oxf})$.

Ungari, C., Filiaci, F., Riccardi, E., Rinna, C., \& Iannetti, G. (2012). Etiology and incidence of zygomatic fracture: a retrospective study related to a series of 642 patients. Eur Rev Med Pharmacol Sci.

Usta, J., Antoun, J., Ambuel, B., \& Khawaja, M. (2012). Envolvendo o sistema de saúde na violência doméstica: o que as mulheres desejam. Ann Fam Med. 